\title{
Johnson Noise Thermometry System Requirements
}

\section{January 2013}

Prepared by

Charles L. Britton Jr.

Michael Roberts

Nora D. Bull

Lou Qualls

David E. Holcomb

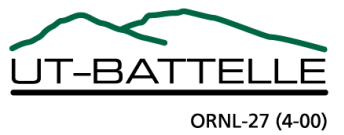




\title{
DOCUMENT AVAILABILITY
}

Reports produced after January 1, 1996, are generally available free via the U.S. Department of Energy (DOE) Information Bridge.

Web site http://www.osti.gov/bridge

Reports produced before January 1, 1996, may be purchased by members of the public from the following source.

\author{
National Technical Information Service \\ 5285 Port Royal Road \\ Springfield, VA 22161 \\ Telephone 703-605-6000 (1-800-553-6847) \\ TDD 703-487-4639 \\ Fax 703-605-6900 \\ E-mail info@ntis.gov \\ Web site http://www.ntis.gov/support/ordernowabout.htm
}

Reports are available to DOE employees, DOE contractors, Energy Technology Data Exchange (ETDE) representatives, and International Nuclear Information System (INIS) representatives from the following source.

Office of Scientific and Technical Information

P.O. Box 62

Oak Ridge, TN 37831

Telephone 865-576-8401

Fax 865-576-5728

E-mail reports@osti.gov

Web site http://www.osti.gov/contact.html

\begin{abstract}
This report was prepared as an account of work sponsored by an agency of the United States Government. Neither the United States Government nor any agency thereof, nor any of their employees, makes any warranty, express or implied, or assumes any legal liability or responsibility for the accuracy, completeness, or usefulness of any information, apparatus, product, or process disclosed, or represents that its use would not infringe privately owned rights. Reference herein to any specific commercial product, process, or service by trade name, trademark, manufacturer, or otherwise, does not necessarily constitute or imply its endorsement, recommendation, or favoring by the United States Government or any agency thereof. The views and opinions of authors expressed herein do not necessarily state or reflect those of the United States Government or any agency thereof.
\end{abstract}


ORNL/TM-2013/XXX

SMR/ICHMI/ORNL/TR-2013/XX

\title{
JOHNSON NOISE THERMOMETRY SYSTEM REQUIREMENTS
}

\author{
Author(s) \\ Charles L. Britton Jr \\ Michael Roberts \\ Nora D. Bull \\ Lou Qualls \\ David E. Holcomb
}

Date Published: January 2013

Prepared by

OAK RIDGE NATIONAL LABORATORY

Oak Ridge, Tennessee 37831-6283

managed by

UT-BATTELLE, LLC

for the

U.S. DEPARTMENT OF ENERGY

under contract DE-AC05-00OR22725 


\section{CONTENTS}

Page

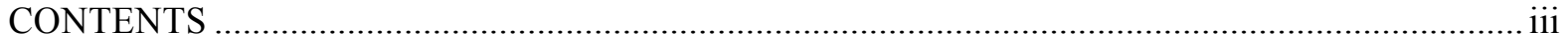

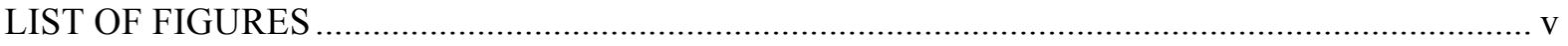

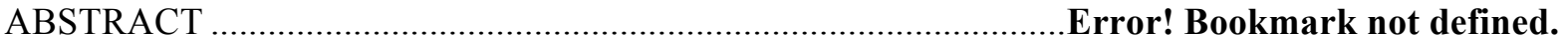

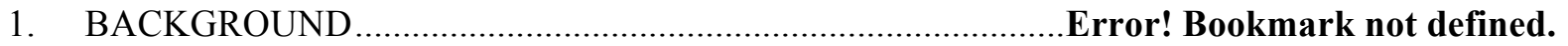

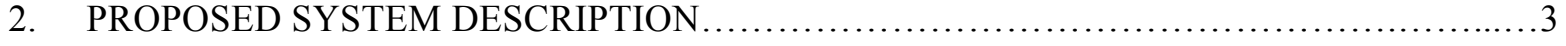

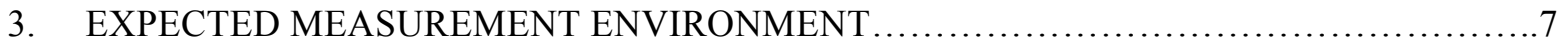

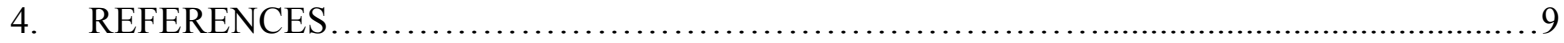




\section{LIST OF FIGURES}

Figure

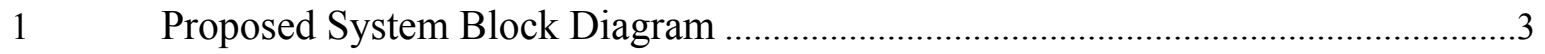

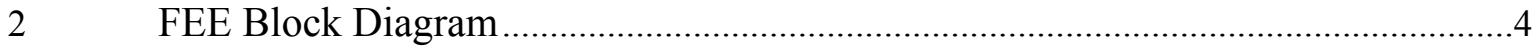

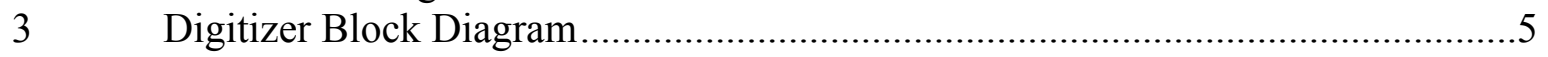

$4 \quad$ Single-ended to differential conversion .................................................. 


\begin{abstract}
This document is intended to capture the requirements for the architecture of the developmental electronics for the ORNL-lead drift-free Johnson Noise Thermometry (JNT) project conducted under the Instrumentation, Controls, and Human-Machine Interface (ICHMI) research pathway of the U.S. Department of Energy (DOE) Advanced Small Modular Reactor (SMR) Research and Development (R\&D) program. The requirements include not only the performance of the system but also the allowable measurement environment of the probe and the allowable physical environment of the associated electronics. A more extensive project background including the project rationale is available in the initial project report [1].
\end{abstract}

\title{
1. BACKGROUND
}

Stable and reliable instrumentation is needed for effective reactor operations. Instrumentation naturally drifts from calibration over time and each channel must be periodically visited, inspected and calibrated. To minimize the impact of random drift, multiple redundant sensor measurements are often taken and averaged at the same physical location. The assumption is that random drifts can both increase or decrease an instrument's response and the average of a significant number will produce representative readings. This strategy increases the overall number of sensors in a plant, which in-turn increases the instrumentation capital and maintenance costs. Also, multiple redundant sensors do not address systematic measurement drift vulnerability.

The power levels proposed in SMR concepts are as much as an order of magnitude lower than that of a traditional nuclear power plant. However, the number of potential plant variables that must be measured does not necessarily decrease with plant power levels. If similar instrumentation strategies are employed in SMRs then the total number of sensors and the costs to install and maintain them will be roughly equivalent, making the relative costs of instrumentation in an SMR higher than that of larger plants. This detracts from the potential economic viability of small reactors.

In order to decrease instrumentation costs in SMRs, the number of sensors installed needs to decrease and/or the allowable interval between maintaining those sensors needs to increase. Because of their high capital costs and (relatively) low fuel costs, nuclear power plants are usually operated as baseload plants at $\sim 100 \%$ of capacity and are maintained such that they remain online during the peak demand periods of the year: summer and winter. Refueling and maintenance outages are therefore scheduled at six-month intervals and large power plants typically extend operating cycles to 18-months.

Extending the operating cycle increases revenue generation relative to operational costs. The Tennessee Valley Authority investigated the maintenance activities for a large pressurized water reactor and determined that most of the activities could either be performed with the reactor online or they could be delayed to permit four years of continuous operation [2]. Some activities, including temperature measurement at critical plant locations, could not be easily extended beyond two years. JNT is a measurement technology that can potentially overcome this limitation to extend operating cycles for SMR systems.

The accuracy of primary loop temperature measurement directly impacts the quantity of power produced, compliance with regulation, and component lifetimes. The technical specifications for nuclear power plants (NPPs) limit their total allowable power output. In 
order to comply with this requirement, the planned power output is reduced from the allowable maximum by the amount of process measurement uncertainty. Temperature measurement in the primary coolant loop along with the coolant mass flow rate provides the plant thermal power measurement. Any potential drift in the primary loop temperature measurement must be accounted for in the operating margin. Hence, decreasing the temperature measurement uncertainty can directly increase the plant revenue. Additionally, one of the contributing factors to the operating temperature limit is the lifetime of the primary loop components. If the plant exceeds the design temperature for a significant period of time, the component lifetime will be reduced necessitating early (and costly) replacement.

Properly operating Resistance Temperature Detectors (RTDs) are among the most accurate industrial temperature measurement technologies available. In the planned implementation, a resistance-based measurement within of the4-wire platinum element the JNT system will provide a prompt temperature measurement. The JNT system serves as a diverse first-principles temperature measurement providing a continuous correction to the resistance to temperature correlation. It offers a fundamental temperature measurement that is both redundant and diverse without requiring an additional instrument sensor. The JNT system has the potential to provide verification that the RTD is functioning properly and provide an in-situ calibration of the RTD. Thus, it could potentially be used to reduce overall sensor count and extend time between sensor channel calibrations. Because of this capability, JNT has been considered as a primary temperature measurement system for long-duration space missions that must operate for more than a decade with no maintenance [3].

RTD temperature methods with JNT capability could be used in one of two scenarios within a SMR. A select few JNT augmented sensors could be used to provide diversity among a larger group of traditional sensors. In this scenario the total number of sensors could potentially be reduced. In an alternate scenario, the majority of the sensors at a critical location could be JNT augmented RTDs. In this case, a continual check of the health of each instrument channel could not only allow fewer sensors to be used but it could also allow an increase in the maintenance interval.

In order for JNT to be beneficial to SMR designers, it must offer advantages beyond the current state-of-the-art technology. Comparisons to traditional RTDs and thermocouples will involve life cycle costs, installation footprint, reliability and accuracy. There is additional equipment beyond what is required for the traditional RTD measurement. Therefore, the JNT-RTD system will involve additional complexity and this additional complexity must be justified. Operators will want to know that the measurement is reliable and trustworthy. It is also important that the sensor involve little, if any, additional work and that it has a low probability of causing malfunction of the primary measurement channel. If these features can be demonstrated, then the JNT-RTD system could potentially save money and increase plant reliability. The JNT-RTD system does not necessarily require improved accuracy over currently used technologies because their accuracy is acceptable for plant operation, and even if the JNT system were to be more accurate it would not be useful if it were less reliable or less cost effective.

The locations that could benefit from the JNT-RTD system are those that are difficult to access due to their physical location and those that cannot be accessed during normal operation because of risks to personnel. These locations will vary for each plant design. Currently the technology is being demonstrated to a nominal distance of $80^{\prime}$, however 
performance will ultimately be distance limited. The length of extension line from the sensor to the front end electronics decreases the sensitivity of the system. This distance will be examined experimentally in order to establish the potential limits of operation and understand how those limits may be affected by design variables such as quality of the extension cables and stability of front end amplifiers. Using this information, plant designers can evaluate where JNT can be used to make their designs safer and more economical.

\section{PROPOSED SYSTEM DESCRIPTION}

The proposed Johnson Noise Thermometer system consists of several primary frontend electronics (FEE) components and signal-processing electronics (SPE) as shown in Fig. 1 .

1. Sensor and interconnect (FEE). The present plan is to use a standard platinum resistance temperature detector (RTD) connected by a durable cable that will be capable of withstanding the high temperatures experienced by the RTD. This cable is likely to be a mineral-insulated type and should be long enough to place the subsequent electronics modules outside of the reactor containment area, a distance of nominally $25 \mathrm{~m}$.

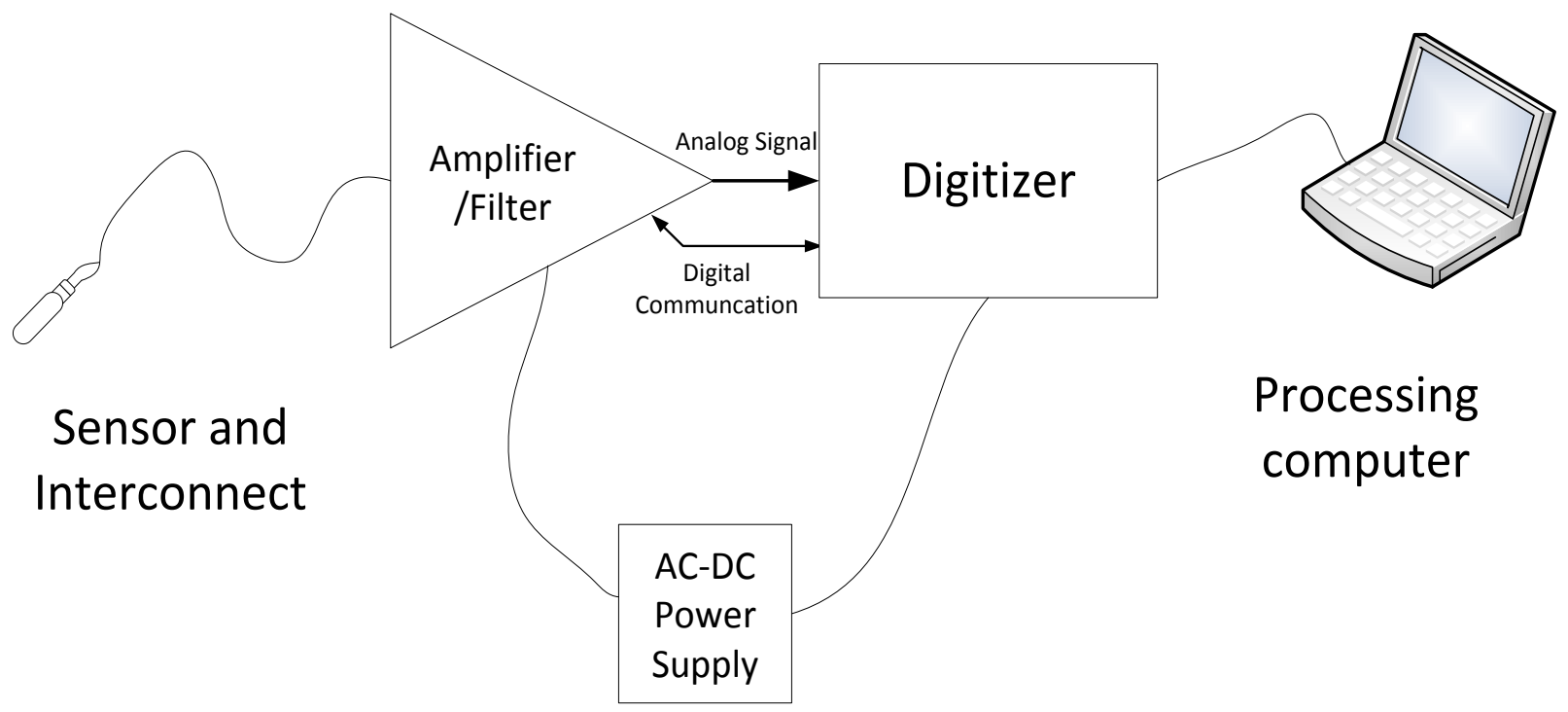

Fig. 1. Proposed System Block Diagram.

2. Amplifier/Filter (FEE). This module processes the noise from the sensor by receiving, amplifying, and filtering the signal. In addition it implements the DC ohmic measurement of the RTD. The swept-tone interface electronics are located here also. In general, any signal associated with the RTD sensor passes through this module. 
3. Digitizer (SPE). The digitizer contains the analog-digital converters (ADCs), digitalanalog converters (DACs), and other control and signal processing functions required for converting the analog signals into computer-readable information for processing.

4. Processing computer (SPE). The processing computer operates the software that collects the data from the digitizer and sends control signals to the amplifier filter. The current software is written in National Instruments Labview and runs under Microsoft Windows.

5. AC-DC Power Supply. An extremely important component of this system is the supply of DC power to the amplifiers and digitizers. This power must be stable and clean (low noise). This is currently intended to be a commercial off-the-shelf supply.

\section{Amplifier/Filter Description}

Figure 2 presents a block diagram of the connectivity of the new system. Each of the two noise channels connects from the RTD sensor via two sets of cables arranged in differential pairs. The preamplifier board contains two identical differential-input JFET preamplifiers/filters/gain sections each with a voltage gain of approximately $80 \mathrm{~dB}$. The system bandwidth is planned to be switchable with low-frequency cutoffs of $500 \mathrm{~Hz}$ and $1 \mathrm{kHz}$. The high-frequency cutoff is planned to be $50 \mathrm{kHz}$ and $100 \mathrm{kHz}$. For the DC resistance measurement (ohmmeter measurement), a 100uA current is supplied to the input and a separate differential amplifier measures the voltage drop across the RTD. This voltage is amplified and filtered. All of these filtered outputs are routed to the digitizer.

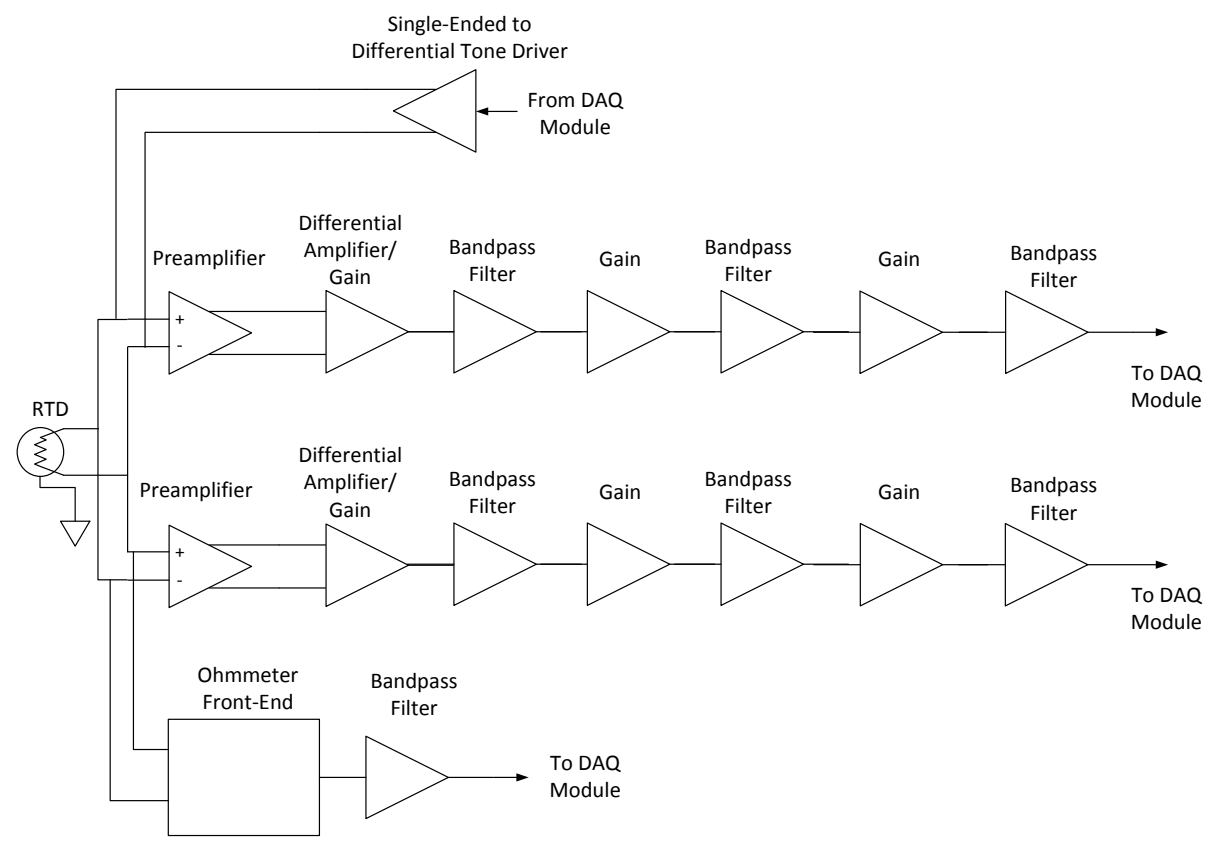

Fig. 2. FEE Block Diagram 
The calibration tone is implemented as a swept sin-wave over the bandwidth of the system. The tone is synthesized on the digitizer board and distributed to the FEE board where it is injected into the input of the preamplifier/RTD. We are currently evaluating whether to inject this tone as a continuous sweep, as multiple discreet frequencies, or to inject white noise.

\section{Digitizer Description}

The digitizer receives the processed analog inputs from the amplifier/filter module and translates them into digital information which is sent to the signal processing program residing on the processing computer. In addition the digitizer acts as a controller which programs and operates the digital waveform synthesizer. The waveform synthesizer generates the pilot tone or random noise input to the processing electronics.

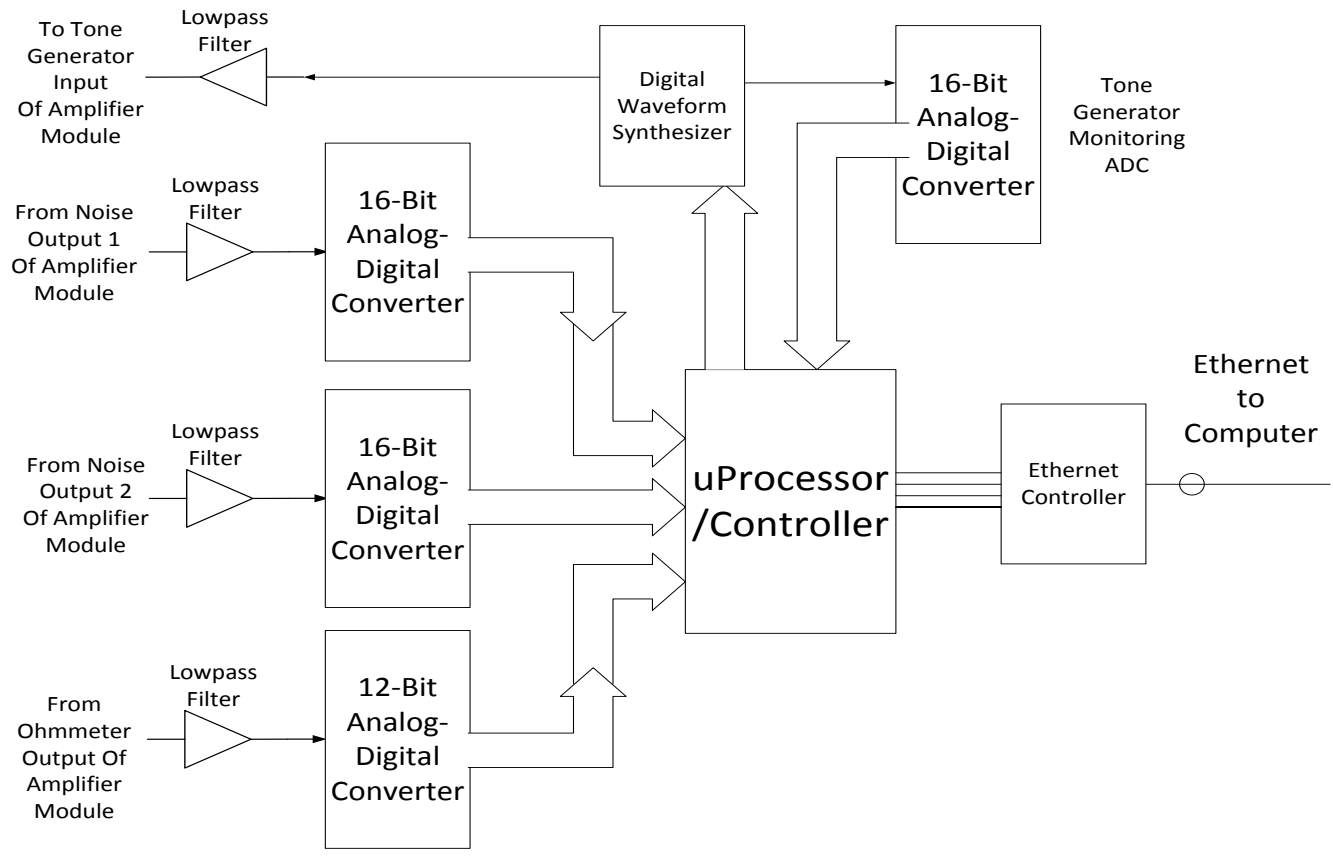

Fig 3. Digitizer Block Diagram

Because there are many commercial options for the ADCs, the form factor of the digitizer will not be decided until later in the project when the actual design begins. The options range from a single board with COTS circuits on the board designed at ORNL to a partially-designed ORNL board with the waveform synthesizer and interface connected to a commercial multi-channel digitizer. The primary considerations will involve the following:

1. Performance - The performance of the combined FEE and digitizer system is the primary consideration for this project.

2. Computer interface - The present software utilizes LabView drivers to interface to the digitizer/data acquisition system. A custom board will require the writing of drivers to interface to the board. The amount of driver creation will be a determining factor when the design cycle for the digitizer system begins. 


\section{Processing Computer/ Interface Description}

The computer used for this system is a Windows-based device running Windows7 or higher. We are currently running on a Dell desktop using LabView as the programming software for the JNT calculations and control and we plan to remain with that platform combination through our present development/demonstration cycle. The final system will utilize Ethernet between the computer and digitizer. We have chosen Ethernet because it is a mature hardware interface that is becoming increasingly available in nuclear power plants. It is not proprietary and will therefore be convenient to install in a variety of facilities.

\section{Software Algorithm Description}

As previously mentioned, the software that performs the processing of the JNT signals as well as the control of the system is implemented in LabView. The actual algorithm is relatively complex so that a summary will be presented here.

The sensor temperature $T_{s}$ can theoretically be computed from knowledge of the sensor resistance $R_{s}$ and the open-circuit noise voltage power spectral density (PSD) $\mathrm{G}_{s}(f)$ of the sensor by the formula

$$
T_{s}=\frac{\mathrm{G}_{s}(f)}{2 k R_{s}}
$$

where $k$ is Boltzmann's constant $1.38 \times 10^{-23}$ and where $\mathrm{G}_{s}(f)$ is constant up to very high frequencies (in the $\mathrm{THz}$ range). We directly measure the sensor resistance very accurately with a four-wire method. We cannot directly measure the sensor's open-circuit noise PSD but we can make a good estimate of it by amplifying the sensor open-circuit noise voltage with two independent low-noise amplifiers and computing their cross power spectral density (CPSD). By averaging multiple estimates of the CPSD we effectively eliminate the contribution of the amplifiers' noise and get a CPSD that is virtually due to the sensor noise alone. We then divide that result by the cross power frequency response of the two amplifiers to estimate the sensor noise PSD. We actually estimate the sensor resistance from the formula

$$
T_{s}=\frac{1}{2 k R_{s}}\left[\frac{\widehat{\mathrm{G}}_{12}(f)}{\widehat{\mathrm{H}}_{12}(f)}-2 k T_{c} R_{c}\right]
$$

in which $\widehat{\mathrm{G}}_{12}(f)$ is our best estimate of the CPSD between the two amplifier output signals, $\widehat{\mathrm{H}}_{12}(f)$ is our best estimate of the cross power frequency response of the two voltage amplifiers, $T_{c}$ is the temperature of the cable connecting the sensor to the amplifiers and $R_{c}$ is the resistance of the cable conductors. The contribution of the cable noise to the CPSD is small but not completely negligible. If the cable resistance were zero or if the cable temperature were at absolute zero, this correction would not be needed.

We also measure the frequency response of the entire system by injecting a known signal we call the "pilot tone" into the sensor. By correlating the amplifier outputs with the known pilot tone we can compute the amplifier frequency responses. This allows continuous monitoring of the amplifier frequency responses to maintain their calibration for long-term operation. The tone generator sections in Figs. 2 and 3 implement this function. 


\section{EXPECTED MEASUREMENT ENVIRONMENT}

\section{Sensor Description}

The sensors we have been using for JNT development are standard 100-ohm Resistance Temperature Detector (RTD) constructed of platinum. Platinum is the standard for resistance thermometers since it has a very wide usable temperature range $(1000 \mathrm{C})$ and is very linear over this range [4]. Platinum resistance thermometers have been extremely well characterized over the years and are widely available from several manufacturers.

The configuration we are using is the standard 4-wire measurement shown in the FEE block diagram of Fig. 2 [5]. This can be an inherently accurate measurement in implemented correctly since it removes the interconnecting cable wire resistance from the DC measurement. It is not, however, ideal for the JNT part of the measurement in that there is twice the cable and therefore twice the capacitance compared to a two-wire measurement. The effect of capacitance will be covered in a later section of this report. In addition, the length of cable we will use has its own small but significant amount of DC resistance. This resistance, like the RTD, generates Johnson noise and must be subtracted from the RTD noise.

The sensor is required to be in a metal sheath that acts as an electrostatic shield. In addition, we currently are planning to electrically insulate the RTD from its surroundings using an alumina or other sheath.

The cable to be used in a JNT system is a critical component. For a permanentlyinstalled system, we would expect to use mineral-insulated (MI) cable with either one or two pairs of wires inside. Mineral-insulated cables are solid-sheath cables with wires inside the sheath and an insulator of magnesium oxide packed in for insulation. These cables can be made to go to very high temperature (in excess of 2000C) and can take high radiation doses. We are currently investigating the potential use of this type of cable.

For the testing phase of this project, the long-term radiation hardness of MI or perhaps the temperature range will not be needed since this will depend upon the facilities available for the testing of the system. The actual cable used for testing will therefore be a decision made late in the second year of this project. The type of cable chosen also involves the connectors to be used on the RTD and on the electronics case.

Sensor Measurement Environment

1. Sensor Temperature Range - Measurement will be made in water with a range from $25^{\circ} \mathrm{C}$ to $800^{\circ} \mathrm{C}$.

2. Sensor Measurement Response Time - Because the actual real-time measurement of temperature is accomplished by the DC measurement of the RTD and not the JNT part of the measurement, the measurement response time will depend entirely on the RTD used for the measurement and the thermal time constants involving the RTD structure. The electronics used in the JNT system for DC measurement will have a bandwidth $(\sim 1 \mathrm{KHz})$ much greater than thermal time constants.

3. Sensor Pressure Environment - TBD.

\section{FEE/SPE Location}


As previously stated, we plan to locate the FEE/SPE modules immediately outside the reactor containment to minimize the RTD interconnect cable length and therefore cable capacitance. This capacitance has a deleterious effect on the RTD signal bandwidth. Because we have a system that utilizes a resistive sensor located some distance from the actual preamplifier input that is used to sense and amplify the noise signal, there will always be some level of bandwidth reduction of the noise signal due to capacitance across the sensor. The effect is simply the single-pole frequency rolloff of the effective noise generator (Pt resistor). Although the actual JNT system has a differential connection to the RTD, we can view the cable/RTD combination as either a differential, balanced system or a singleended system. It is relatively easy to show that the two forms shown in Fig. 4 are equivalent. The time constant of either circuit in Fig. 4 is given by

$$
\tau_{h i}=R * C
$$

The 3-dB voltage frequency of the circuit in Fig. 4 is given by

$$
f_{h i}=\frac{1}{2 \pi * R * C}
$$

For a $100 \Omega$ resistor with a temperature coefficient of $0.00385 \Omega / \Omega /{ }^{\circ} \mathrm{C}$, the value at $800 \mathrm{C}$ will be $375 \Omega$. Assuming our final cable will have similar characteristics to the microphone cable we are currently using, the capacitance of the cable $\mathrm{C}$ is $7.9 \mathrm{nF}$. The system $3 \mathrm{~dB}$ bandwidth is therefore is approximately $54 \mathrm{kHz}$.
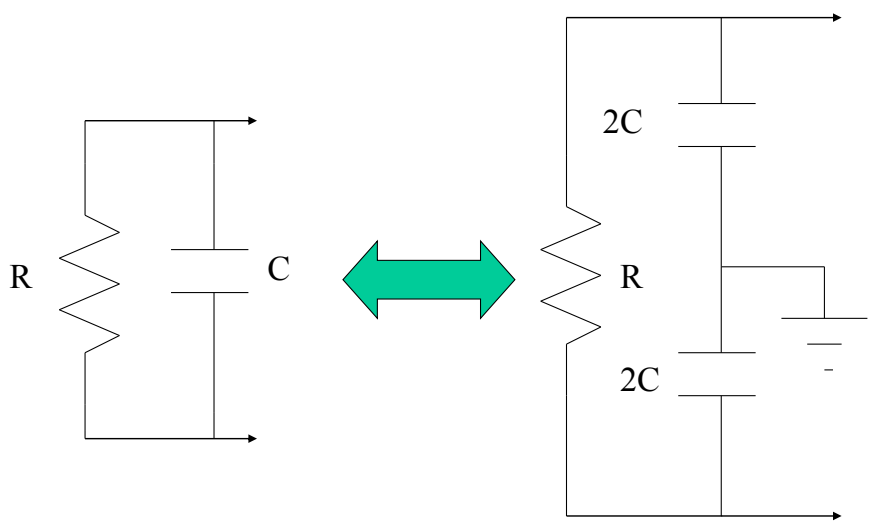

Fig. 4. Single-ended to differential conversion.

The spot noise (noise power at a particular frequency) of the above circuit can be found by taking the real part of the impedance as

$$
\bar{e}^{2}=4 k T \Delta f * \operatorname{Re}\{Z\}
$$

where $\mathrm{Z}$ is the impedance of the above network. This can be found to be 


$$
\bar{e}^{2}(f)=4 k T \Delta f * R * \frac{1}{1+\frac{f^{2}}{f_{h i}^{2}}}
$$

This describes the reduction in available noise power as a function of frequency. At frequencies above the pole of the circuit, we lose noise power at a rate of $40 \mathrm{~dB} / \mathrm{decade}$ of frequency.

After the printed circuit board for the FEE is finalized, we will be able to size the required box in which the FEE board and SPE board will be housed.

\section{FEE / Size Constraints}

Currently there are no projected size constraints identified. The system will be made as small and compact as practical.

\section{FEE/SPE Operating Temperature}

The standard commercial electronics operating temperature range is $-40 \mathrm{C}$ to $+85 \mathrm{C}$. All components going into the design and fabrication of these modules will be at least the commercial operating range. We plan to operate the circuitry at a maximum of $+45 \mathrm{C}$. Environment surrounding the electronics will be from $25^{\circ} \mathrm{C}$ to $40^{\circ} \mathrm{C}$ with up to $95 \% \mathrm{RH}$. The equipment is not currently required to survive in a condensing atmosphere or submerged.

\section{FEE/SPE Interference Tolerance}

There is presently no requirement for the interference or vibration rejection for this system but either or both of these can interfere with the measurement. One of the key components of the JNT software is that of interference rejection. We are presently not clear as to how to quantify these as a hard specification and intend to report them as we test the final system.

[1] ORIGINAL REPORT

\section{REFERENCES}

[2] FIND REFERENCE

[3] SP-100 or JIMO

[4] Hashemian, H. N., Sensor Reliability and Performance, Instrumentation Systems and Automation Society, 2005, ISBN 1-55617-932-4 , p.12

[5] Ibid Hashemian, p.23 